\title{
Lions, tigers, and bears, oh sh!t: Semantics versus tabooness in speech production
}

\author{
Katherine K. White ${ }^{1} \cdot$ Lise Abrams $^{2} \cdot$ Sarah M. Koehler ${ }^{1} \cdot$ Richard J. Collins $^{1}$
}

Published online: 6 June 2016

(C) Psychonomic Society, Inc. 2016

\begin{abstract}
While both semantic and highly emotional (i.e., taboo) words can interfere with speech production, different theoretical mechanisms have been proposed to explain why interference occurs. Two experiments investigated these theoretical approaches by comparing the magnitude of these two types of interference and the stages at which they occur during picture naming. Participants named target pictures superimposed with semantic, taboo, or unrelated distractor words that were presented at three different stimulus-onset asynchronies (SOA): $-150 \mathrm{~ms}, 0 \mathrm{~ms}$, or $+150 \mathrm{~ms}$. In addition, the duration of distractor presentation was manipulated across experiments, with distractors appearing for the duration of the picture (Experiment 1) or for $350 \mathrm{~ms}$ (Experiment 2). Taboo distractors interfered more than semantic distractors, i.e., slowed target naming times, at all SOAs. While distractor duration had no effect on type of interference at -150 or 0 SOAs, briefly presented distractors eliminated semantic interference but not taboo interference at +150 SOA. Discussion focuses on how existing speech production theories can explain interference from emotional distractors and the unique role that attention may play in taboo interference.
\end{abstract}

Keywords Speech production - Picture-word interference . Semantic interference $\cdot$ Taboo interference

\section{Katherine K. White}

whitek@rhodes.edu

1 Department of Psychology, Rhodes College, 2000 North Parkway, Memphis, TN 38112, USA

2 Department of Psychology, University of Florida, Gainesville, FL, USA
Speaking involves selecting the appropriate words to communicate an intended message and is typically rapid and accurate (Levelt, 1989). However, as with any cognitive process, speaking is susceptible to interference from other words present at the time of production. Interference from semantically related words during production has been well-established using a picture-word interference (PWI) task (Schriefers, Meyer, \& Levelt, 1990; Starreveld \& La Heij, 1996). Participants are asked to name pictures quickly and accurately that are superimposed with distractor words; producing a picture name (tiger) is slowed when a distractor word is semantically related (lion) compared with unrelated (bowl). Interference from highly emotional distractors, specifically taboo words, also has been shown to slow word production during PWI, although only a few studies have investigated this phenomenon (Dhooge \& Hartsuiker, 2011; White, Abrams, LaBat, \& Rhynes, 2016). Although both semantic and taboo distractors can interfere with speech production, different theoretical mechanisms are thought to underlie the resulting interference. The purpose of the present experiments was to investigate these mechanisms by directly comparing the magnitude and stage (i.e., time point) of semantic and taboo interference during speech production.

It is widely assumed that word production requires at least two stages (Dell, 1986; Levelt, Roelofs, \& Meyer, 1999): before articulation, speakers select the lexical representation that best communicates an idea and then proceed to map speech sounds onto (i.e., phonologically encode) that representation. However, theories diverge on the locus of semantic interference in speech production. According to competitive models of lexical selection (e.g., WEAVER++, Roelofs, 1992), the lexical representations for the distractor and picture name compete for retrieval during lexical selection. The WEAVER++ model maintains that semantic distractors exceed a competition threshold, a type of attentional filter, and 
then directly compete for selection with the target (Piai, Roelofs, \& Schriefers, 2012). Semantic distractors also may be more difficult to reactively block, a process that attenuates distractor influence so that the picture can be named (Roelofs, 2003). Alternatively, noncompetitive models (Finkbeiner \& Caramazza, 2006; Mahon et al., 2007) ascribe a post-lexical locus of interference: Because distractors are read before forming a response to the picture, distractors enter an output buffer before the picture name. The bottleneck nature of the output buffer requires the distractor to be removed from the buffer to name the picture. Items in the buffer are checked for accuracy by a control process, recently proposed to be the verbal self-monitor (Dhooge \& Hartsuiker, 2012). The monitor is sensitive to several criteria, such as shared attributes between the target and distractor, and thus removal of the distractor takes longer when it is from the same semantic category as the picture (e.g., animal).

Can these two explanations for semantic interference during PWI also be applied to taboo interference? Under competitive models, distractor characteristics (e.g., visibility; Piai et al., 2012) are proposed to influence the likelihood that distractors exceed the competition threshold. We propose that a distractor's emotional characteristics, specifically its tabooness, affect the rate at which distractors exceed the threshold: Tabooness allows distractors to more rapidly exceed the competition threshold due to their high arousal and speeded lexical access (Keuper et al., 2012), drawing attentional resources away from picture naming and slowing naming times. Evidence also suggests that disengaging from strong emotional words is difficult (Frings, Englert, Wentura, \& Bermeitinger, 2010), which could result in slower blocking of taboo distractors. Noncompetitive models explain taboo interference by defining social inappropriateness as a criterion to which the self-monitor is sensitive. Taboo words are inappropriate to speak (particularly in experimental settings), so they are carefully monitored and more slowly removed from the output buffer, slowing picture naming times.

Although the occurrence of taboo interference can be explained within existing theoretical mechanisms, these mechanisms do not specify the degree to which taboo distractors will interfere or whether all types of distractors interfere similarly. Previous studies suggest that semantic distractors typically slow picture naming by approximately $30-50 \mathrm{~ms}$ (Damian \& Martin, 1999; Mahon et al., 2007; Starreveld \& La Heij, 1996). In contrast, the few studies that have investigated slowing from taboo distractors have demonstrated varied effect sizes, ranging from $38 \mathrm{~ms}$ (Dhooge \& Hartsuiker, 2011) to more than $100 \mathrm{~ms}$ (White et al., 2016). Therefore, taboo distractors may or may not be more disruptive to picture naming than semantic distractors.

Clarifying this pattern has implications for both competitive and noncompetitive models of speech production by illustrating whether the continued engagement of attention by taboo distractors makes blocking them more difficult or slows their removal from the output buffer, relative to nonemotional distractors.

The theoretical explanations for taboo interference could be further refined by determining the stage(s) at which a taboo distractor interferes with speech production. We applied a method that has traditionally been used to determine the locus of semantic interference: manipulating the stimulus-onset-asynchrony (SOA) between distractor and target presentation. For example, semantic interference is typically observed when the picture and distractor are presented simultaneously ( $0 \mathrm{SOA}$ ) or the distractor is presented at a negative SOA, i.e., 100-200 ms before the picture (Damian \& Martin, 1999; Starreveld \& La Heij, 1996), suggesting interference during lexical selection (although interference at later SOAs can sometimes occur; Damian \& Martin, 1999). If the semantic distractor is presented at a positive SOA more than $200 \mathrm{~ms}$ after picture onset, interference is unlikely to occur because retrieval of the picture's lexical representation is complete and phonological encoding has begun. While previous studies using taboo distractors have only used simultaneous presentation of the picture and distractor, the theoretical underpinnings of taboo interference suggest that it may be less constrained to specific stages of speech production than semantic interference. Because taboo distractors do not share any inherent relation with the to-be-produced picture names, their ability to interfere is not constrained to the stage of production when the picture's lexical representation is retrieved (as is the case for semantic distractors within competitive models) and could interfere at any SOA.

We compared the size of semantic and taboo interference by presenting target pictures superimposed with semantic, taboo, or unrelated (neutral) distractor words. The stage(s) at which interference occurs also were measured by presenting distractors at three SOAs: $150 \mathrm{~ms}$ before, $0 \mathrm{~ms}$ (simultaneously), or $150 \mathrm{~ms}$ after picture onset.

\section{Experiment 1}

\section{Method}

\section{Participants}

Forty native English-speaking undergraduates (29 females; $M=18.92$ years, $S D=1.10$ ) participated in exchange for partial course credit.

\section{Materials}

Target pictures A set of 27 target pictures were selected with the provision that no more than five targets came from a 
Table 1 Example target pictures and their assigned semantic, taboo, and unrelated distractors

\begin{tabular}{llll}
\hline Target picture & Semantic distractor & Taboo distractor & Unrelated distractor \\
\hline Asparagus & Celery & Orgasm & Trinity \\
Bus & Train & Crap & Joke \\
Duck & Goose & Breasts & Clown \\
Glove & Mitten & Dildo & Citrus \\
Elbow & Hand & Fuck & Game \\
\hline
\end{tabular}

particular semantic category. Target pictures were 3.5 " x 3.5 " black drawings on a white background chosen from Google images.

Distractors Targets were each assigned one taboo, one semantic, and one unrelated distractor whose primary part of speech was noun (see Table 1 for examples). Semantic distractors were category coordinates taken from previous studies (Costa et al., 2005; Damian \& Martin, 1999; Mahon et al., 2007), and taboo distractors were selected from taboo norms (Janschewitz, 2008). Semantic, taboo, and unrelated distractors did not differ in word frequency (as measured by HAL $\log$ and SUBTLEX $\log$ ), word length, and orthographic neighborhood (Balota, Yap, Cortese, Hutchison, Kessler, et al., 2007), $p \mathrm{~s}>0.17$ (Table 2). A post-experiment rating task showed that participants rated the three types of distractor words as equally familiar, $p \mathrm{~s}>0.88$.

Fillers Fifty-four filler pictures were included to reduce carryover effects from taboo target trials (White et al., 2016) but were not analyzed. Filler pictures were accompanied by a simultaneously presented, unrelated distractor word. Filler pictures and distractors were neutral in valence $\left(M_{\text {pictures }}=\right.$ $\left.5.34, M_{\text {distractors }}=5.29\right)$ and low in arousal $\left(M_{\text {pictures }}=3.67\right.$, $\left.M_{\text {distractors }}=3.59\right)$ (Bradley \& Lang, 1999; Janschewitz, 2008).

\section{Procedure}

Participants were first familiarized with the appropriate names for each picture. Each target and filler picture was randomly presented (without any distractor), and participants provided a name for each picture. Pictures that were named incorrectly were re-presented until all of the correct picture names were produced.

Following five practice trials, participants were instructed to name each picture as quickly and accurately as possible while ignoring the distractor word. Before each picturedistractor trial, a question mark appeared and remained on screen until the space bar was pressed. A fixation (+) then appeared for $500 \mathrm{~ms}$ and was immediately replaced with a picture and/or superimposed distractor. Distractors appeared $150 \mathrm{~ms}$ before ( $-150 \mathrm{SOA})$, simultaneously with (0 SOA), or $150 \mathrm{~ms}$ after (+150 SOA) the target. Consistent with White et al. (2016), the picture and word remained on the screen until either the space bar was pressed or $3000 \mathrm{~ms}$ elapsed. Naming times (in $\mathrm{ms}$ ) were extracted from wavefiles using a voice onset program (Jennings \& Abrams, 2016). Pictures were presented in counterbalanced blocks so that each picture was named with one distractor type (taboo, semantic, or unrelated) in each of three blocks. Distractor SOA also was counterbalanced so that each distractor type was presented with one SOA in each block.

Target pictures were followed by two filler pictures, randomly selected and presented once in each block. Following
Table 2 Means (and standard deviations) of lexical characteristics matched across distractors

\begin{tabular}{cccccc}
\hline & $\begin{array}{l}\text { HAL log } \\
\text { frequency }\end{array}$ & $\begin{array}{l}\text { SUBTLEX log } \\
\text { frequency }\end{array}$ & $\begin{array}{l}\text { Word length (\# } \\
\text { letters })\end{array}$ & OrthoN & $\begin{array}{l}\text { Familiarity } \\
\text { rating }\end{array}$ \\
\hline $\begin{array}{c}\text { Taboo } \\
\text { distractors }\end{array}$ & $7.94^{\mathrm{a}}(1.46)$ & $2.49^{\mathrm{a}}(.62)$ & $5.48(1.63)$ & $5.24^{\mathrm{a}}(5.59)^{\mathrm{a}}$ & $4.87(1.52)$ \\
$\begin{array}{c}\text { Semantic } \\
\text { distractors }\end{array}$ & $7.71(1.82)$ & $2.46(.73)$ & $5.63(1.55)$ & $4.15(4.94)$ & $4.93(1.23)$ \\
$\begin{array}{c}\text { Unrelated } \\
\text { distractors }\end{array}$ & $8.37(1.69)$ & $2.43(.69)$ & $5.63(1.62)$ & $3.74(4.46)$ & $4.92(1.43)$ \\
\hline
\end{tabular}

OrthoN refers to orthographic neighborhood

${ }^{\mathrm{a}}$ These means are based on only 21 of the 27 taboo distractors that have frequencies listed in the English Lexicon Project (Balota et al., 2007) database. 
completion of the experiment, participants rated each distractor on valence, arousal, and familiarity using 1-9 Likert scales, with higher values representing more positive, more arousing, and more often encountered, respectively.

\section{Results and discussion}

All wavefiles were checked manually for accuracy, and any naming times that were not easily determined by the program were manually coded $(5.7 \%)$. Trials were excluded from analyses when a wavefile failed to record or was undecipherable, and when participants made speech errors, failed to respond within $3000 \mathrm{~ms}$, or produced the wrong picture name (3.3\%). Outliers, calculated as naming times greater or less than $2.5 S D$ from each participant's/item's mean, were removed ( $3.4 \%$ of trials)

Log-transformed naming times were submitted to a linear mixed-effects analysis using the lmerTest (Kuznetsova, Brockhoff, \& Christensen, 2015) supplement to the lme4 package (Bates, Maechler, Bolker, \& Walker, 2014). ${ }^{1}$ Fixed effects were distractor type and SOA, and random intercepts were included for participants and items. ${ }^{2}$ There were 1,329 data observations. There were two questions of theoretical interest. First, we compared the size of the interference effects and showed that taboo interference was larger than semantic interference: Naming times were slower when distractors were taboo $(M=958 \mathrm{~ms})$ than when they were semantic ( $M=862 \mathrm{~ms}, t=7.77, p<0.001)$, which in turn were slower than unrelated distractors $(M=823 \mathrm{~ms}, t=2.43, p=0.015)$. The second question of theoretical interest was whether the degree of taboo interference (i.e., difference between taboo and unrelated conditions) and the degree of semantic interference (i.e., difference between semantic and unrelated conditions) changed as a function of SOA (Fig. 1). We first tested the 3 (Distractor Type) x 3 (SOA) interaction, which was significant, $\chi^{2}(2)=13.31, p<0.001$. Then, to assess taboo interference, separate 2 (Distractor Type: Taboo, Unrelated) x 2 (Distractor SOA) mixed-effect regression models were tested for each pair of SOAs. These analyses showed that taboo interference was equivalent at $-150 \mathrm{SOA}$ and $0 \mathrm{SOA}$ and then decreased at +150 SOA. Specifically, less taboo interference occurred at +150 SOA compared with -150 SOA, $t=3.25, p=$ 0.001 , and compared with $0 \mathrm{SOA}, t=3.35, p<0.001$, whereas -150 and 0 SOA did not differ, $t=0.18, p=0.86$. Comparable 2 (Distractor Type: Semantic, Unrelated) x 2 (Distractor SOA)

\footnotetext{
${ }^{1}$ Analyses using repeated measures analysis of variance by participants $\left(F_{1}\right)$ and items $\left(F_{2}\right)$ revealed the same pattern of results and significant effects as the linear mixed-effects tests.

${ }^{2}$ Although Barr et al. (2013) recommended fitting the maximal model with random intercepts and slopes, doing so created an overly-specific model that failed to converge, suggesting that the model was too complex for the data. In these cases, Bates and colleagues (2015) recommend a more parsimonious model, which is reported here.
}

mixed-effect regression models showed that the degree of semantic interference was similar at all SOAs: -150 compared with $0, t=0.83, p=0.41,-150$ compared with $+150, t=0.24$, $p=0.81$, and 0 compared with $+150, t=1.18, p=0.24$.

The finding that taboo interference was greater than semantic interference demonstrates the robustness of the taboo interference effect. The size of the taboo interference effect as shown in Fig. 1 replicates White et al. (2016), who presented distractors at 0 SOA, and extends the taboo interference effect to both -150 and +150 SOAs. The results also suggest that the two types of distractors differ somewhat in the stage at which they interfere, with the taboo but not semantic interference effect decreasing at later stages of production $(+150 \mathrm{SOA})$. However, these findings may be contingent on the long presentation duration of distractors; Dhooge and Hartsuiker (2011) found a smaller taboo interference effect and used shorter presentation duration of distractors $(350 \mathrm{~ms})$. Furthermore, there is evidence that distractor duration affects whether semantic distractors interfere at later stages of production: Damian and Martin (1999) found semantic interference at +200 SOA when distractors remained on the screen with the picture but not when distractors were only briefly presented $(200 \mathrm{~ms})$. Thus, Experiment 2 attempted to replicate Experiment 1 using distractor duration of $350 \mathrm{~ms}$, consistent with Dhooge and Hartsuiker (2011).

\section{Experiment 2}

\section{Method}

\section{Participants}

Forty participants ( 23 females; $M=19.1$ years, $S D=1.1$ ) were compensated as in Experiment 1.

\section{Materials}

The materials were identical to Experiment 1 with the exception that two target picture names and eight filler picture names were changed after participants commented on unintentional visual similarities between some pictures used in Experiment 1.

\section{Procedure}

The only change in procedure was that distractors were terminated $350 \mathrm{~ms}$ after their onset.

\section{Results and discussion}

Naming times (in ms) were treated as in Experiment 1, with $22.7 \%$ of trials manually coded and $2.5 \%$ of trials excluded 


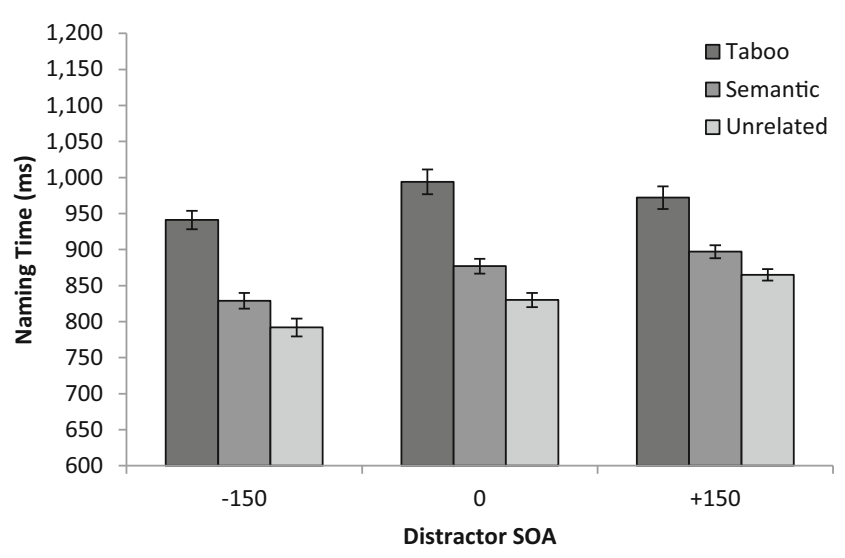

Fig. 1 Mean picture naming time (ms) in Experiment 1 as a function of distractor SOA and distractor type. Interference effects can be computed by comparing the unrelated mean with each of the distractor type's means. Error bars represent one standard error from the mean

for recording or speech errors. Of the remaining correct trials, analyses excluded trials where naming times were greater or less than $2.5 S D$ from each participant's mean ( $4.2 \%$ of trials).

Data analysis procedures were identical to Experiment $1 .^{3}$ There were 1,329 data observations. Similar to Experiment 1, naming times were slower when distractors were taboo $(M=1,129 \mathrm{~ms})$ than when distractors were semantic ( $M=1,046 \mathrm{~ms}, t=9.94, p<0.001$ ), which were slower than when distractors were unrelated ( $M=998 \mathrm{~ms}, t=2.05, p=0.04)$. As in Experiment 1, the 3 (Distractor Type) $\mathrm{x} 3$ (SOA) interaction was significant, $\chi^{2}(2)=24.48, p<0.001$. A series of separate 2 (Distractor Type) x 2 (Distractor SOA) mixed-effect regression models were tested to determine if the degree of interference changed as a function of SOA (Fig. 2). Similar to Experiment 1 , taboo interference was greater at the earlier SOAs compared with the later SOA: Interference differed for $-150 \mathrm{SOA}$ and +150 SOA, $t=4.91, p<0.001$, and for 0 SOA and +150 $\mathrm{SOA}, t=2.16, p=0.03$. Different from Experiment 1 was the finding of greater taboo interference at -150 SOA compared to 0 SOA, $t=2.71, p=0.01$. In contrast to taboo interference, the degree of semantic interference was equivalent at -150 and 0 SOAs, $t=0.58, p=0.56$ but disappeared at +150 . No semantic interference occurred at +150 SOA compared with -150 SOA, $t=2.41, p=0.02$, and 0 SOA, $t=2.77, p=0.01$.

The present results provide an important replication of the taboo interference effect, which was similar across both experiments even though the overall naming times in Experiment 2 were slower than those in Experiment 1. Taboo words are particularly disruptive to speech production, regardless of their presentation duration. This interference occurs even when distractors appear relatively late in the production process. In

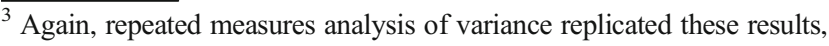
except for the difference in taboo interference at -150 and 0 SOAs, which was significant only in the item analysis.
}

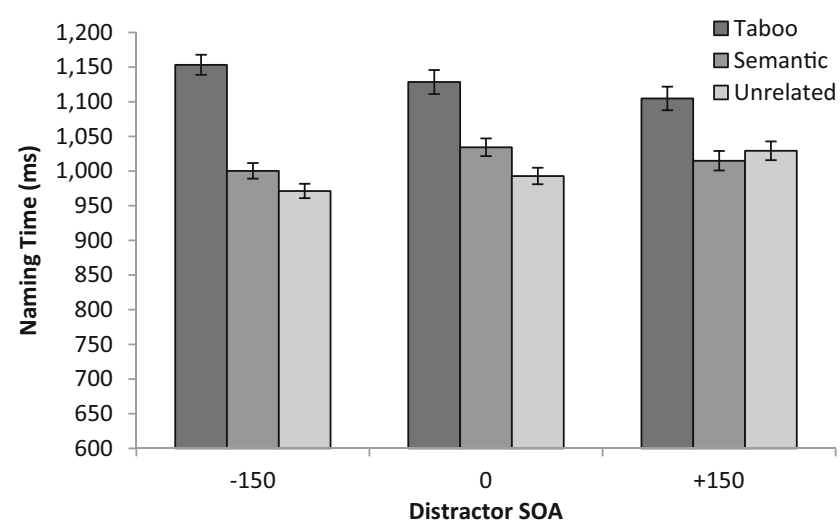

Fig. 2 Experiment 2's mean picture naming time (ms) as a function of distractor SOA and distractor type. Interference effects can be computed by comparing the unrelated mean with each of the distractor type's means. Error bars represent one standard error from the mean

contrast, reducing distractor duration isolated semantic interference to -150 and $0 \mathrm{~ms}$ SOAs, consistent with the assumption that semantic distractors interfere immediately before or during access of the picture's lexical representation (Damian \& Martin, 1999). These results suggest that different mechanisms underlie semantic versus taboo interference, and the implications of this are discussed in more detail below.

\section{General discussion}

These experiments showed that all distractors do not interfere equally. Compared with semantic distractors, taboo distractors produced greater slowing effects during picture naming and occurred at all SOAs and both distractor durations, suggesting that taboo words interfere with picture naming at all stages of word production. These findings extend previous research by more thoroughly establishing the magnitude of taboo interference as larger than semantic interference effects and documenting the stages at which this interference is strongest.

First, manipulating distractor SOA allowed us to establish that unlike semantic distractors, taboo distractors disrupt lexical selection as well as later stages of target retrieval, specifically phonological encoding and articulation. The timing of interference is unique to taboo distractors, because interference from semantic distractors did not occur at $+150 \mathrm{~ms}$ SOA when distractors were presented for brief durations. Although there was a reduction in taboo interference from earlier SOAs to later SOAs, this is due to an unexpected increase in naming times when distractors were unrelated, not a reduced naming time when distractors are taboo. ${ }^{4}$ Nonetheless, the occurrence of taboo interference at all SOAs and both distractor durations demonstrates a ubiquity that does not occur for semantic interference.

\footnotetext{
${ }^{4}$ Although unclear why naming time might increase for unrelated distractors presented at $+150 \mathrm{SOA}$, this finding has been present in other studies (Sailor, Brooks, Bruening, Seiger-Gardner, \& Guterman, 2009).
} 
Second, the duration of distractor presentation had little effect on the size of taboo interference, as taboo distractors consistently slowed picture naming by more than $100 \mathrm{~ms}$. The size of taboo interference in Experiment 1 replicates White et al. (2016) for distractors presented for the duration of picture presentation. We also replicated the typical effect size of semantic interference (approximately $40 \mathrm{~ms}$ in both experiments), suggesting that our semantic interference was an appropriate baseline for comparison with the taboo interference effect. However, the taboo effect size in Experiment 2 for briefly presenting distractors was larger than that found by Dhooge and Hartsuiker (2011), possibly because our experiment used neutral filler trials following taboo trials, which Dhooge and Hartsuiker did not have. These filler trials are relevant because naming of a filler trial is slowed when it follows a taboo target trial compared to an unrelated/neutral trial (White et al., 2016). Consequently, the size of the taboo interference effect found in Dhooge and Hartsuiker may have been an underestimate; The mean naming time of their neutral trials may have been slowed by taboo carryover effects.

How are these findings accommodated within existing models of speech production? Under competitive models, two attentional mechanisms have been proposed to regulate a distractor's influence on picture naming and are thus candidates to explain taboo interference. Evidence in support of the competition threshold's role in taboo interference comes from finding taboo interference at a positive SOA, where distractors should not interfere once lexical selection of the target has been initiated, unless attention can be rapidly engaged by the distractor. Taboo distractors must therefore quickly grab attention, consistent with a faster rate of exceeding the competition threshold, which in turn slows picture naming. It is plausible that highly arousing emotional words more rapidly exceed a competition threshold than semantically related words, in part because the former are quickly recognized (Keuper et al., 2012) and/or because taboo words have lower thresholds for detection (Gaillard et al., 2006). It is worth noting an alternative explanation for why taboo, but not semantic, distractors interfered at later SOAs: The strength of taboo distractors may be greater than semantic distractors, allowing them to exert their influence throughout the entire time course of lemma access, whereas semantic distractors can only exert their influence earlier, when lemma access is at its peak. However, White et al. (2016) demonstrated the existence of carryover effects following taboo trials, i.e., slowing on a subsequent trial following a trial with a taboo distractor, which is problematic for this explanation because lemma access from the previous trial has already been completed. The second mechanism, blocking, also is likely to affect the size of interference effects. Because taboo distractors continue to engage attention relative to other types of distractors, blocking them is more difficult and results in larger interference effects than other distractor types. Finding taboo interference at an early SOA, coupled with White et al.'s (2016) demonstration of taboo carryover effects, suggests that disengagement from taboo distractors may be particularly difficult.

The idea that there are two mechanisms within competitive models that regulate taboo interference parallels evidence from emotional Stroop studies, where interference from strong emotional words may result from two sources: the rapid and automatic allocation of attention, and engagement of a slower, more controlled threat-detection mechanism (Frings et al., 2010). It is worth noting that the relation between the competition threshold and the blocking mechanism is relatively unexplored. Our results suggest that emotional properties of distractors, such as their tabooness, may affect both mechanisms, i.e., not only how quickly they are blocked but also the rate at which they exceed the threshold. Future research should more precisely specify the independent and interactive contributions of each of these mechanisms.

Unlike competitive models, noncompetitive models explain both semantic and taboo interference in terms of greater time to remove distractors from the output buffer, which is consistent with slower picture naming times following the presence of either type of distractor. However, these models may need further refinement to accommodate greater taboo interference effects relative to semantic interference, such as conditions under which distractor criteria require additional monitoring; It is not clear why it would take longer to remove a taboo versus a semantic distractor. With respect to the stages at which taboo words interfere, our findings are inconsistent with positing removal of distractors from the output buffer as the sole source of taboo interference. First, it is unclear why taboo interference at $-150 \mathrm{~ms} \mathrm{SOA}$ was equivalent (in Experiment 1) or larger (in Experiment 2) than at $0 \mathrm{~ms}$ SOA, as earlier-presented distractors should have had more time to be removed from the buffer. Second, although a reduction in taboo interference at +150 SOA is consistent with these models (because target retrieval is initiated prior to distractor presentation), it is not clear why there is any taboo interference at $+150 \mathrm{SOA}$ if the target enters the buffer first.

\section{Conclusions}

These experiments extend our knowledge about the ways in which distractor words impede the ability to produce language, one of our most automatic and fundamental skills. Our findings suggest that all distractors are not equally distracting: Highly emotional, taboo words interfere more than semantically related words despite the former having no relationship to the to-be-produced speech. Furthermore, unlike semantic interference, interference from taboo distractors occurs at later stages of word retrieval, demonstrating a robustness that is relatively independent of distractor duration or time point at which the distractor is introduced. Future 
research should continue to document various sources in which emotion can interfere with speaking, such as a speaker's emotional state (e.g., anxiety). Doing so would offer a more comprehensive and ecological approach to understanding the dynamic nature of speech production.

Acknowledgments The authors thank Anne Hohlt and Lisa Hsi for assistance with data collection and coding and Henry Hua for assistance with statistical analyses. Portions of this research were presented at the $54^{\text {th }}$ annual meeting of the Psychonomic Society and the $27^{\text {th }}$ annual convention of the Association for Psychological Science.

\section{References}

Balota, D. L., Yap, M. J., Cortese, M. J., Hutchison, K. A., Kessler, B., Loftis, B., ... Treiman, R. (2007). The English Lexicon Project. Behavior Research Methods, 39, 445-459. doi:10.3758/ BF03193014

Barr, D. J., Levy, R., Scheepers, C., \& Tily, H. J. (2013). Random effects structure for confirmatory hypothesis testing: Keep it maximal. Journal of Memory and Language, 68, 255-278. doi:10.1016/j. jm1.2012.11.001

Bates, D., Kliegl, R., Vashishth, S., \& Baayen, H. R. (2015). Parsimonious mixed models. arXiv:1506.04967.

Bates, D., Maechler, M., Bolker, B., \& Walker, S. (2014)._lme4: Linear mixed-effects models using Eigen and S4_. R package version 1.17, URL: http://CRAN.R-project.org/package=lme4

Bradley, M. M., \& Lang, P. J. (1999). Affective norms for English words (ANEW): Stimuli, instruction manual and affective ratings, Technical report $C$-1. Gainesville, FL: The Center for Research in Psychophysiology, University of Florida.

Costa, A., Alario, F. -X., \& Caramazza, A. (2005).On the categorical nature of the semantic interference effect in the picture-word interference paradigm. Psychonomic Bulletin \& Review, 12, 125-131.

Damian, M. F., \& Martin, R. C. (1999). Semantic and phonological codes interact in single word production. Journal of Experimental Psychology: Learning, Memory, and Cognition, 25, 345-361. doi: 10.1037/0278-7393.25.2.345

Dell, G. S. (1986). A spreading-activation theory of retrieval in sentence production. Psychological Review, 93, 283-321. doi:10.1037/0033295X.93.3.283

Dhooge, E., \& Hartsuiker, R. J. (2011). How do speakers resist distraction? Evidence from a taboo picture-word interference task. Psychological Science, 22, 855-859. doi:10.1177/ 0956797611410984

Dhooge, E., \& Hartsuiker, R. J. (2012). Lexical selection and verb selfmonitoring: Effects of lexicality, context, and time pressure in picture-word interference. Journal of Memory and Language, 66, 163-176. doi:10.1016/j.jml.2011.08.004

Finkbeiner, M., \& Caramazza, A. (2006). Now you see it, now you don't: On turning semantic interference into facilitation in a Stroop-like task. Cortex, 42, 790-796. doi:10.1016/S0010-9452(08)70419-2
Frings, C., Englert, J., Wentura, D., \& Bermeitinger, C. (2010). Decomposing the emotional Stroop effect. The Quarterly Journal of Experimental Psychology, 63, 42-49. doi:10.1080/ 17470210903156594

Gaillard, R. I., Del Cul, A., Naccache, L., Vinckier, F., Cohen, L., \& Dehaene, S. (2006). Nonconscious semantic processing of emotional words modulates conscious access. PNAS, 103, 7524-7529.

Janschewitz, K. (2008). Taboo, emotionally valenced, and emotionally neutral word norms. Behavior Research Methods, 4, 1065-1074. doi:10.3758/BRM.40.4.1065

Jennings, D. T., \& Abrams, L. (2016). Automatic detection of voice onset times from waveform data using Microsoft Excel. Manuscript in preparation.

Keuper, K., Zwanzger, P., Nordt, M., Eden, A., Laeger, I., Zwitserlood, P., ... Dobel, C. (2012). How 'love' and 'hate' differ from 'sleep': Using combined electro/magnetoencephalographic data to reveal the sources of early cortical responses to emotional words. Human Brain Mapping, 35, 875-888. doi:10.1002/hbm.22220

Kuznetsova, A., Brockhoff, P. B., \& Christensen, R. H. B. (2015). lmerTest: Tests in Linear Mixed Effects Models. R package version 2.0-25. http://CRAN.R-project.org/package=lmerTest

Levelt, W. J. M. (1989). Speaking: From intention to articulation. Cambridge, MA: MIT Press.

Levelt, W. J. M., Roelofs, A., \& Meyer, A. S. (1999). A theory of lexical access in speech production. Behavioral and Brain Sciences, 22, 175. doi: $10.1017 / \mathrm{S} 0140525 X 99001776$

Mahon, B. Z., Costa, A., Peterson, R., Vargas, K. A., \& Caramazza, A. (2007). Lexical selection is not by competition. Journal of Experimental Psychology: Learning, Memory, and Cognition, 33, 503-533. doi:10.1037/0278-7393.33.3.503

Piai, V., Roelofs, A., \& Shriefers, H. (2012). Distractor strength and selective attention in picture-naming performance. Memory and Cognition, 40, 614-627. doi:10.3758/s13421-011-0171-3

Roelofs, A. (1992). A spreading-activation theory of lemma retrieval in speaking. Cognition, 42, 107-142. doi:10.1016/0010-0277(92) 90041-F

Roelofs, A. (2003). Goal-referenced selection of verbal action: Modeling attentional control in the Stroop task. Psychological Review, 110, 88-125. doi:10.1037/0033-295X.110.1.88

Sailor, K., Brooks, P. J., Bruening, P. F., Seiger-Gardner, L., \& Guterman. M. (2009). Exploring the time course of semantic interference and associative priming in the picture-word interference task. The Quarterly Journal of Experimental Psychology, 62, 789-801. doi: 10.1080/17470210802254383

Schriefers, H., Meyer, A. S., \& Levelt, W. J. M. (1990). Exploring the time course of lexical access in language production: Picture-word interference studies. Journal of Memory and Language, 29, 86-102. doi:10.1016/0749-596X(90)90011-N

Starreveld, P. A., \& La Heij, W. (1996). Time-course analysis of semantic and orthographic context effects in picture naming. Journal of Experimental Psychology: Learning, Memory, and Cognition, 22, 869-918. doi:10.1037/0278-7393.22.4.896

White, K. K., Abrams, L., LaBat, L. R., \& Rhynes, A. M. (2016). Competing influences of emotion and phonology during pictureword interference. Language, Cognition and Neuroscience, 31, 265-283. doi:10.1080/23273798.2015.1101144 